\title{
First Results of GERDA Phase II
}

\author{
(Gerda Collaboration) \\ M. Agostini ${ }^{a}$, M. Allardt ${ }^{d}$, A.M. Bakalyarov ${ }^{m}$, M. Balata ${ }^{a}$, I. Barabanov ${ }^{k}$, L. Baudis ${ }^{s}$, C. Bauer ${ }^{g}$, \\ E. Bellotti ${ }^{h, i}$, S. Belogurov ${ }^{l, k}$, S.T. Belyaev ${ }^{m}$, G. Benato ${ }^{s}$, A. Bettini ${ }^{p, q}$, L. Bezrukov ${ }^{k}$, T. Bode $^{o}$, \\ D. Borowicz ${ }^{c, e}$, V. Brudanin $e$, R. Brugnera ${ }^{p, q}$, A. Caldwell ${ }^{n}$, C. Cattadori ${ }^{i}$, A. Chernogorov $^{l}$, \\ V. D’Andrea*a, E.V. Demidova ${ }^{l}$, N. Di Marco ${ }^{a}$, A. Domula ${ }^{d}$, E. Doroshkevich ${ }^{k}$, V. Egorov $^{e}$, \\ R. Falkenstein ${ }^{r}$, N. Frodyma $^{c}$, A. Gangapshev ${ }^{k, g}$, A. Garfagnini ${ }^{p, q}$, C. Gooch ${ }^{n}$, P. Grabmayr ${ }^{r}$, \\ V. Gurentsov ${ }^{k}$, K. Gusev ${ }^{e, m, o}$, J. Hakenmüller ${ }^{g}$, A. Hegai ${ }^{r}$, M. Heisel $^{g}$, S. Hemmer ${ }^{q}$, W. Hofmann ${ }^{g}$, \\ M. Hult ${ }^{f}$, L.V. Inzhechik ${ }^{k}$, J. Janicskó Csáthy ${ }^{o}$, J. Jochum ${ }^{r}, \mathbf{M}$. Junker $^{a}$, V. Kazalov ${ }^{k}, \mathbf{T}_{\text {Kihm }}{ }^{g}$, \\ I.V. Kirpichnikov ${ }^{l}$, A. Kirsch ${ }^{g}$, A. Kish ${ }^{s}$, A. Klimenko ${ }^{g, e}$, R. Kneiß| ${ }^{n}$, K.T. Knöpfle ${ }^{g}$, O. Kochetov ${ }^{e}$, \\ V.N. Kornoukhov ${ }^{l, k}$, V.V. Kuzminov ${ }^{k}$, M. Laubenstein ${ }^{a}$, A. Lazzaro ${ }^{o}$, V.I. Lebedev ${ }^{m}$, B. Lehnert ${ }^{d}$, \\ H.Y. Liao ${ }^{n}$, M. Lindner ${ }^{g}$, I. Lippi ${ }^{q}$, A. Lubashevskiy ${ }^{g}, e$, B. Lubsandorzhiev ${ }^{k}$, G. Lutter ${ }^{f}$, \\ C. Macolino ${ }^{a}$, B. Majorovits ${ }^{n}$, W. Maneschg ${ }^{g}$, E. Medinaceli ${ }^{p, q}$, M. Miloradovic ${ }^{s}$, R. Mingazheva ${ }^{s}$, \\ M. Misiaszek ${ }^{c}$, P. Moseev ${ }^{k}$, I. Nemchenok ${ }^{e}$, D. Palioselitis ${ }^{n}$, K. Panas ${ }^{c}$, L. Pandola ${ }^{b}$, K. Pelczar $^{c}$, \\ A. Pullia ${ }^{j}$, S. Riboldi ${ }^{j}$, N. Rumyantseva ${ }^{e}$, C. Sada ${ }^{p, q}$, F. Salamida ${ }^{i}$, M. Salathe ${ }^{g}$, C. Schmitt $^{r}$, \\ B. Schneider ${ }^{d}$, S. Schönert ${ }^{o}$, J. Schreiner ${ }^{g}$, O. Schulz ${ }^{n}$, A.-K. Schütz ${ }^{r}$, B. Schwingenheuer ${ }^{g}$, \\ O. Selivanenko $^{k}$, E. Shevzik ${ }^{e}$, M. Shirchenko ${ }^{e}$, H. Simgen ${ }^{g}$, A. Smolnikov ${ }^{g, e}$, L. Stanco $^{q}$, \\ L. Vanhoefer ${ }^{n}$, A.A. Vasenko ${ }^{l}$, A. Veresnikova ${ }^{k}$, K. von Sturm ${ }^{p, q}$, V. Wagner ${ }^{g}$, A. Wegmann ${ }^{g}$, \\ T. Wester ${ }^{d}$, C. Wiesinger ${ }^{o}$, M. Wojcik ${ }^{c}$, E. Yanovich ${ }^{k}$, I. Zhitnikov ${ }^{e}$, S.V. Zhukov ${ }^{m}$, D. Zinatulina ${ }^{e}$, \\ K. Zuber ${ }^{d}$, and G. Zuzel ${ }^{c}$. \\ $\left.{ }^{a}\right)$ LNGS \& GSSI (INFN), ${ }^{b}$ ) LNS (INFN), ${ }^{c}$ ) IoP Cracow, ${ }^{d}$ ) TU Dresden, ${ }^{e}$ ) JINR Dubna, ${ }^{f}$ ) EU, \\ Geel, ${ }^{g}$ ) MPI Heidelberg, $\left.{ }^{h}\right)$ U. Bicocca Milan, ${ }^{i}$ ) INFN Bicocca Milan, ${ }^{j}$ ) U.d.Studi Milan, \\ ${ }^{k}$ ) INR Moscow, ${ }^{l}$ ) ITEP Moscow, ${ }^{m}$ ) Kurchatov Moscow, ${ }^{n}$ ) MPI Munich, ${ }^{o}$ ) TUM Munich, ${ }^{p}$ ) U. \\ Padova, ${ }^{q}$ ) INFN Padova, ${ }^{r}$ ) EKUT Tübingen, ${ }^{s}$ ) U. Zurich \\ E-mail: gerda-eb@mpi-hd.mpg •de
}

The GErmanium Detector Array (GERDA) experiment, located at the Laboratori Nazionali del Gran Sasso, searches for the $0 v \beta \beta$ decay of ${ }^{76}$ Ge. GERDA Phase II was started in December 2015 , aiming to reach a sensitivity on the $0 v \beta \beta$ decay half-life larger than $10^{26} \mathrm{yr}$ in three $\mathrm{yr}$ of data taking with about $100 \mathrm{~kg} \cdot \mathrm{yr}$ exposure with background index of about $10^{-3} \mathrm{cts} /(\mathrm{keV} \cdot \mathrm{kg} \cdot \mathrm{yr})$. The major upgrade of Phase II is the deployment of thirty newly produced Broad Energy Germanium detectors. They contribute to the background reduction with better energy resolution and enhanced pulse shape discrimination. To achieve the necessary background reduction, the setup was complemented with LAr veto. After 6 months of operation a first data release with $10.8 \mathrm{~kg} \cdot \mathrm{yr}$ exposure was performed, showing that the background goal has been achieved. A new lower limit on the $0 v \beta \beta$ decay half life of ${ }^{76} \mathrm{Ge}$ of $5.3 \cdot 10^{25} \mathrm{yr}(90 \%$ C.L.) has been set.

Neutrino Oscillation Workshop

4 - 11 September, 2016

Otranto (Lecce, Italy)

\footnotetext{
* Speaker.
} 

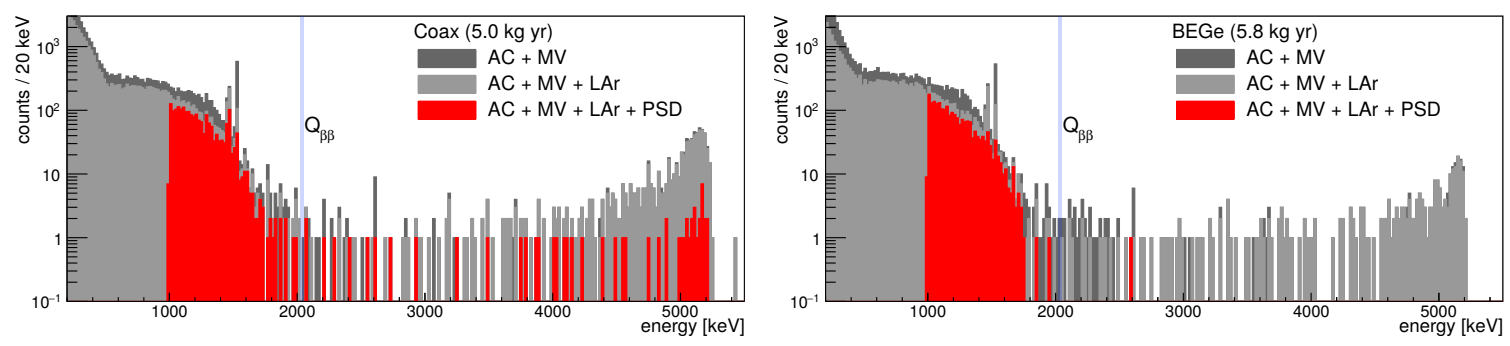

Figure 1: Energy spectrum of first Phase II release for coaxial (left) and BEGe (right) detectors. The darkgray spectra are obtained after anti-coincidence (AC) and muon veto (MV) cuts, the light-gray ones include the liquid argon veto (LAr), in red are reported the final spectra after the pulse shape discrimination (PSD).

\section{The GERDA Experiment}

The GERDA setup [1] has been designed to minimize the main background sources which affected the previous generation experiments. High Purity Germanium (HPGe) detectors enriched to about $87 \%$ in the double beta emitter ${ }^{76} \mathrm{Ge}$ are operated bare in liquid argon (LAr), being both source and detectors of $0 v \beta \beta$ decay. The argon cryostat is complemented by a water tank with $10 \mathrm{~m}$ diameter which further shields from neutron and $\gamma$ backgrounds, also working as muon veto.

A first physics data taking campaign (Phase I) was carried out from November 2011 to June 2013 and the data showed no indication of a $0 v \beta \beta$ signal [2]. After the completion of Phase I the GERDA setup has been upgraded to perform its next step (Phase II): the goal was the tenfold reduction of the background with simultaneous increase of the ${ }^{\text {enr }} \mathrm{Ge}$ detector mass. Thirty Broad Energy Germanium (BEGe) detectors from Canberra [3] were deployed: they allow for superior background rejection and have an excellent energy resolution. In addition an active suppression of background by detecting the LAr scintillation light has been introduced [4]. The LAr veto consists of PMTs and wavelength shifting fibers coupled to SiPMs. Also detectors holders, contacts and front-end electronics have been redesigned to minimize the mass and improve the radiopurity of the materials close to the detector array.

\section{First Phase II results}

On December 20th, 2015 the Phase II data taking started with 40 detectors (30 BEGe, $7{ }^{\mathrm{enr}} \mathrm{Ge}$ coaxial and $3{ }^{\text {nat }} \mathrm{Ge}$ coaxial). The first Phase II data were released after 6 months of data taking, corresponding to an exposure of $10.8 \mathrm{~kg} \cdot \mathrm{yr}$ of ${ }^{\mathrm{enr}} \mathrm{Ge}\left(5.0 \mathrm{~kg} \cdot \mathrm{yr}\right.$ from ${ }^{\mathrm{enr}} \mathrm{Ge}$ coaxial and $5.8 \mathrm{~kg} \cdot \mathrm{yr}$ from BEGe detectors). During the data taking an energy region of $50 \mathrm{keV}$ around $Q_{\beta \beta}$ was blinded.

Fig. 1 shows the Phase II spectra separately for coaxial (left) and BEGe (right) detectors. The prominent features of the spectra are due to the same background components of Phase I [5]. The light grey spectra in Fig. 1 show the effect of the LAr veto (i.e. a factor $\sim 5$ reduction at $\mathrm{K}$ lines and $\sim 2$ in the range 1940-2140 keV), then the background is further reduced by applying the pulse shape discrimination (PSD) cut (red spectra in Fig. 1). The PSD for BEGe detectors is based on the ratio of the peak amplitude of the current signal $A$ and the total energy $E$. Low values of $A / E$ are typical for multi-site events and high values for surface events. For the coaxial detectors the PSD is based on two neural networks to discriminate multi-site and degraded $\alpha$ events. 

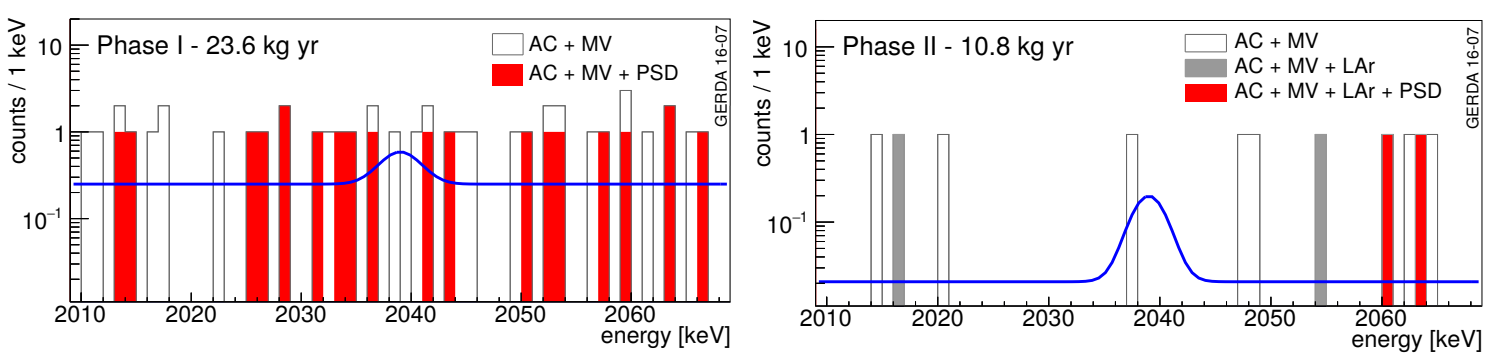

Figure 2: Zoom on the region around $Q_{\beta \beta}$. Left: Phase I data, right: first Phase II data. The abbreviations are the same of Fig. 1. The blue lines show fitted background level and 90\% C.L. limit on $0 v \beta \beta$ decay.

The blinded events in the $Q_{\beta \beta}$ region were processed after fixing all data selection criteria and analysis parameters. Fig. 2 shows the final spectra in the blinded region: Phase I data $(23.6 \mathrm{~kg} \cdot \mathrm{yr})$ and new Phase II data (10.8 kg.yr).

The most important result of Phase II is the achieved background level: in the window of $Q_{\beta \beta} \pm 100 \mathrm{keV}$ only a few events are found in the two datasets, corresponding to a background index of $0.7_{-0.5}^{+1.1} \cdot 10^{-3} \mathrm{cts} /(\mathrm{keV} \cdot \mathrm{kg} \cdot \mathrm{yr})$ for BEGe detectors and $3.5_{-1.5}^{+2.5} \cdot 10^{-3} \mathrm{cts} /(\mathrm{keV} \cdot \mathrm{kg} \cdot \mathrm{yr})$ for coaxial detectors. Fig. 2 (right) shows that in vicinity of $Q_{\beta \beta}$ only two events survived all the cuts: the closest is at $20 \mathrm{keV}$ away from $Q_{\beta \beta}$. These results show how the challenging background goal of Phase II has been achieved: GERDA is the first background free experiment of the field.

A frequentist analysis was performed with a two-side profile likelihood as test statistic [6] combining Phase I data and the new data. The best fit yielded no $0 v \beta \beta$ signal, setting a new limit on the ${ }^{76} \mathrm{Ge} 0 v \beta \beta$ decay half-life of $5.3 \cdot 10^{25} \mathrm{yr}(90 \%$ C.L.). The result is close to the median sensitivity of $4.0 \cdot 10^{25} \mathrm{yr}(90 \%$ C.L.).

\section{Conclusions}

The first data from Phase II showed the quality of the GERDA design and the effectiveness of background suppression techniques, consisting in the powerful pulse shape discrimination of BEGe detectors and the detection of the argon scintillation light. In three years of data taking the background level will lead to a sensitivity for the $0 v \beta \beta$ decay up to $10^{26} \mathrm{yr}$.

Acknowledgement: The GERDA experiment is supported financially by BMBF, DFG, INFN, MPG, NCN, RFBR, and SNF. The institutions acknowledge also internal financial support.

\section{References}

[1] Gerda Collaboration, Eur. Phys. J. C 732330 (2013).

[2] Gerda Collaboration, Phys. Rev. Lett. 111122503 (2013).

[3] Gerda Collaboration, Eur. Phys. J. C 7539 (2015).

[4] Janicskó-Csáthy J. et al. (Preprint 2016).

[5] Gerda Collaboration, Eur. Phys. J. C 742764 (2014).

[6] Cowan G. et al., Eur. Phys. J. C 711554 (2011). 